\title{
Revision of the genus Hoplocrotaphus Telenga, 1950 (Hymenoptera, Braconidae, Opiinae)
}

\author{
Francisco Javier Peris-Felipo', Sergey A. Belokobylskij², \\ Hossein Ali Derafshan ${ }^{3}$, Ehsan Rakhshani ${ }^{3}$
}

I Bleichestrasse 15, CH-4058 Basel, Switzerland 2 Zoological Institute, Russian Academy of Sciences, St Petersburg, 199034, Russia; Museum and Institute of Zoology, Polish Academy of Sciences, Wilcza 64, Warszawa 00-679, Poland 3 Department of Plant Protection, College of Agriculture, University of Zabol, Zabol, P.O. Box: 98615-538, I. R. Iran

Corresponding author: Francisco Javier Peris-Felipo (peris.felipo@gmail.com)

Academic editor: G. Broad | Received 25 October 2017 | Accepted 13 December 2017 | Published 26 February 2018

http://zoobank.org/FA0E2D73-B517-4915-BF8E-86AA598B40F8

Citation: Peris-Felipo FJ, Belokobylskij SA, Derafshan HA, Rakhshani E (2018) Revision of the genus Hoplocrotaphus Telenga, 1950 (Hymenoptera, Braconidae, Opiinae). Journal of Hymenoptera Research 62: 55-72. https://doi. org/10.3897/jhr.62.21858

\begin{abstract}
A revision of the Palaearctic genus Hoplocrotaphus Telenga, 1950 (Braconidae, Opiinae) is provided; H. hamooniae sp. n., is described and both sexes are illustrated. This genus is recorded for the first time in Iran and Turkmenistan. Illustrated redescriptions of all species are given. An identification key to the species is included.
\end{abstract}

\section{Keywords}

Fly parasitoids, Ichneumonoidea, new species, new records, key, Iran, South Palaearctic

\section{Introduction}

The subfamily Opiinae contains more than 2000 described species worldwide (Yu et al. 2016). Opiinae are strictly koinobiont parasitoids of larvae of Diptera - Cyclorrhapha (Wharton 1999) mainly in leaf mines and fruits. The known hosts of about 300 opiine 
species belong predominantly to the families Agromyzidae, Anthomyiidae and Tephritidae (Fischer 1971, 1972, 1977, 1987; Shaw and Huddleston 1991; Yu et al. 2016).

The genus Hoplocrotaphus Telenga, 1950 is a small (only three known species) and rarely collected taxon with a South Palaearctic distribution (Yu et al. 2016). The main characters to diagnose the genus are: marginal cell of fore wing very short, vein 1-R1 of fore wing in males thickened, occipital carina widely interrupted dorsally (as in the most part of Opiinae taxa) and far separated from hypostomal carina and not fused with it below near mandible, and face below antennal bases lacking tubercles (Tobias 1986).

According to Telenga (1936) one of the important characters of this genus should be presence of transverse ridge on the temples. However, the revision of the holotype material used for the description of $H$. mongolicus Fischer, 1971, H. mysteriosus Fischer, 1971 and $H$. pospelovi Telenga, 1950 showed that only $H$. mongolicus presented such kind of ridge although when type species was checked it looks more as wide subvertical prominence in lower half than a real ridge (Fig. 3E, F). Unfortunately, type specimens of the $H$. pospelovi are without heads (Fig. 7A) and it is impossible to check the real situation with this feature. But according to three conspecific specimens (two males and one female) collected in Turkmenistan in the similar natural condition not far from the species type locality, prominence on temple are completely absent (Fig. 8B, F).

Moreover, it is important to underline the existing incongruence with the species H. mysteriosus because of Fischer (1971) included the presence of "ridge" on the temple in the original description. However, when the same specimen used for the description was revised any prominence could be distinguished (Figs 5B-C, 6A).

For the first time Hoplocrotaphus is reported from Iran and Turkmenistan. In the present paper a new species, $H$. hamooniae sp. n., is described and illustrated. In addition, illustrated redescriptions of all Hoplocrotaphus species and an identification key to species are provided.

\section{Material and methods}

The sampling surveys were carried out during 2015 and 2016 around the Hamoon wetlands in the Sistan region (Iran), where Tamarix stricta Boiss is the dominant species among the opportunistic salt-tolerant trees and shrubs.

The specimens were collected by light trapping; the trap consists of an incandescent bulb and an actinic fluorescent tube placed on a white sheet. The specimens were sampled by hand and directly emerged in $75 \%$ ethanol for subsequent studies. In addition, specimens were collected on Tamarix with a modified sweep net. Afterwards, the specimens were chemically treated according to the AXA method (van Achterberg 2009), mounted on triangular cards and labeled.

For the terminology of morphological features, sculpture and measurements see Peris-Felipo et al. (2014); for wing venation nomenclature see van Achterberg (1993). The following abbreviations are additionally used: POL - postocellar line; OOL - 
ocular-ocellar line; OD - maximum diameter of lateral ocellus. Material was imaged using a Keyence ${ }^{\oplus}$ VHX-2000 Digital Microscope and Adobe Photoshop ${ }^{\oplus}$ software.

The studied specimens, including the types of $H$. hamooniae, are deposited in the collections of the Entomological Collection at the University of Valencia (Spain; ENV), Department of Plant Protection, Faculty of Agriculture, University of Zabol (Iran; DPPZ), Hungarian Natural History Museum (Budapest, Hungary; HNHM) and Zoological Institute RAS (St Petersburg, Russia; ZISP).

\section{Taxonomy part}

\section{Class Hexapoda Blainville, 1816 \\ Order Hymenoptera Linnaeus, 1758 \\ Family Braconidae Nees, 1811 \\ Subfamily Opiinae Leach, 1815 \\ Tribe Opiini Leach, 1815}

\section{Genus Hoplocrotaphus Telenga, 1950}

Type species. Hoplocrotaphus pospelovi Telenga, 1950.

Diagnosis. Head (dorsal view) transverse, with rounded temples behind eye. Ocelli weakly enlarged, arranged in triangle with base larger than its sides. Eyes virtually glabrous. Occipital carina widely interrupted dorsally, in the middle and below (lateral view) complete, not fused with hypostomal carina, usually widely separated from hypostomal carinae and independently reached lower margin of head capsule (Figs 1C, 8B). Clypeus moderately narrow and transverse; hypoclypeal cavity present, but narrow. Mandible rather narrow, usually evenly widened towards its base; its upper tooth longer than lower tooth. First flagellar segment of antenna as long as or weakly longer than second segment. Mesosoma short and high. Notauli mainly absent, finely developed on vertical anterior part. Mesoscutal pit always absent. Prescutellar depression (scutellar sulcus) with numerous carinae. Precoxal suture present, smooth, not reaching anterior and posterior margins of mesopleuron. Propodeum completely smooth. Propodeal spiracles relatively small. Hind femur thickened. Hind tibia weakly widened towards apex, shorter than hind tarsus. Pterostigma of fore wing subtriangular, usually rather wide and short. Marginal cell of fore wing strongly shortened, ending far from apex of wing; vein 1-R1 of fore wing thickened (especially in males), not reaching wing apex and shorter than pterostigma length. Vein SR1 of fore wing evenly curved or sometimes sinuate, in male thickened apically. Vein $r$ of fore wing shorter than pterostigma and broad, in male usually thickened. Second submarginal cell of fore wing rather short. Vein m-cu of fore wing distinctly postfurcal, rarely interstitial. First subdiscal cell of fore wing open apically. First metasomal tergite short, widened towards apex, mainly smooth. Ovipositor more or less exerted. 


\section{Hoplocrotaphus hamooniae Peris-Felipo, Belokobylskij \& Rakhshani, sp. n. http://zoobank.org/CE163734-59B1-437F-B37E-AC63077E0A61 \\ Figures 1, 2}

Material examined. Holotype: + , IRAN: Sistan-o Baluchestan Province, Zabol- Hamoon wetland, $31^{\circ} 09^{\prime} 03.5^{\prime \prime} \mathrm{N}, 61^{\circ} 20^{\prime} 47.04^{\prime} \mathrm{E}, 477 \mathrm{~m}$, 24.ix.2016, light trap, Nim 245 (H.A. Derafshan leg.) (ENV).

Paratypes. $2+$, same data as holotype (DPPZ); 29 , same data as holotype but 20.ix.2016, Nim 241 (ZISP) and 13.x.2016 Nim 250 (DPPZ); 19, same data as holotype but $31^{\circ} 09^{\prime} 03.5^{\prime \prime N}, 61^{\circ} 22^{\prime} 46.8^{\prime \prime E}, 450 \mathrm{~m}$, 19.v.2015, swept on Tamarix stricta L., Nim 84 (H.A. Derafshan leg.) (DPPZ); 19, same data but, 19.iv.2015, Nim 59 (DPPZ). 1 ${ }^{\Uparrow}$, same data as holotype but 20.ix.2016, Nim 101, swept on Tamarix sp. (DPPZ).

Comparative diagnosis. This new species resembles Hoplocrotaphus mongolicus Fischer, 1971, but differs from it in having the first metasomal tergite 0.6-0.8 times as long as its apical width ( 1.3 times in $H$. mongolicus), vein 3-SR 0.8 times as long as vein 2-SR (1.3 times in $H$. mongolicus), clypeus 3.7 times as wide as high (6.6 times in $H$. mongolicus), temple in lower half without prominence (Figs 1C, D, 2A) (with distinct prominence on the temple in $H$. mongolicus: Fig. 3B, E, F), occipital carinae widely separated from hypostomal carina (Fig. 1C) (occipital carinae not strongly separated from hypostomal carina in $H$. mongolicus: Figs 3B, 4A), first flagellar segment 2.4 times as long as its maximum width (3.1 times in $H$. mongolicus) and colour of the body light brown (from dark brown to black in H. mongolicus).

Description. Female (holotype).

Head. In dorsal view, 1.9 times as wide as median length, 1.1 times as wide as mesoscutum, smooth. Eye in lateral view 1.6 times as high as wide and 1.4 times as wide as temple medially. POL 2.3 times OD; OOL 1.7 times OD. Face 2.4 times as wide as high; inner margins of eyes subparallel. Clypeus 3.7 times as wide as high, slightly curved ventrally. Mandible weakly and evenly widened towards base. Temple in lower half without prominence. Occipital carina ventrally widely separated from hypostomal carinae. Antenna 20-segmented. Scape 1.8 times as long as pedicel. First flagellar segment 2.4 times as long as its apical width, as long as second segment; second segment 2.2 times; third to sixth segments 2.0 times, seventh to ninth segments 1.8 times; 10 th to 14 th 1.5 times, 15 th and 16th 1.3 times; 17 th 1.1 times and 18th (apical) segment 2.1 times as long as their maximum width accordingly.

Mesosoma. In lateral view, 1.2 times as long as high. Mesoscutum 0.9 times as long as its maximum width. Posterior mesopleural furrow smooth.

Legs. Hind femur 3.3 times as long as its maximum width. Hind tibia about 6.0 times as long as its maximum subapical width, 0.8 times as long as hind tarsus. First segment of hind tarsus 1.7 times as long as second segment.

Wings. Length of fore wing 2.3 times its maximum width. Marginal cell 3.3 times as long as its maximum width. Vein 1-R1 0.3 times as long as pterostigma, 0.3 times as long as distance between apex of marginal cell and apex of wing. Veins $r$ shorter than 

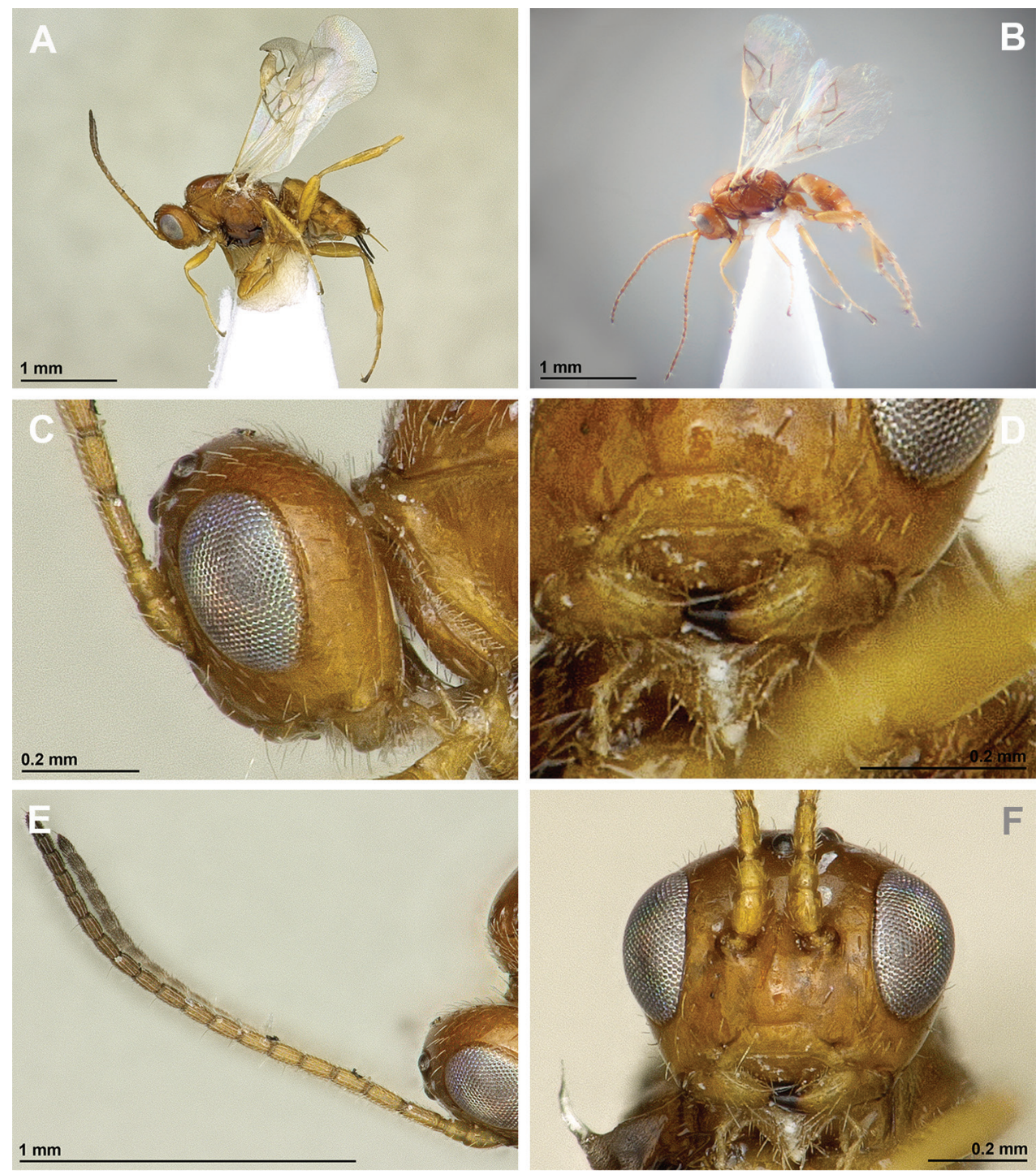

Figure I. Hoplocrotaphus hamooniae sp. n. (A, C-F female holotype B male paratype) A-B Habitus, lateral view C Head, lateral view D Mandible E Antenna F Head, frontal view.

pterostigma broad. Vein 3-SR 0.8 times as long as vein 2-SR. Vein m-cu interstitial. Hind wing 3.9 times as long as its maximum width.

Metasoma. First tergite weakly widened towards apex, 0.6 times as long as its apical width. Ovipositor exerted, 0.9 times as long as first tergite, 0.6 times as long as hind femur.

Length. Body $2.1 \mathrm{~mm}$; fore wing $2.1 \mathrm{~mm}$; hind wing $1.7 \mathrm{~mm}$.

Colour. Body, mandible and legs light brown. Antenna light brown in basal part and dark brown in apical part. Wings hyaline. Pterostigma brown. 

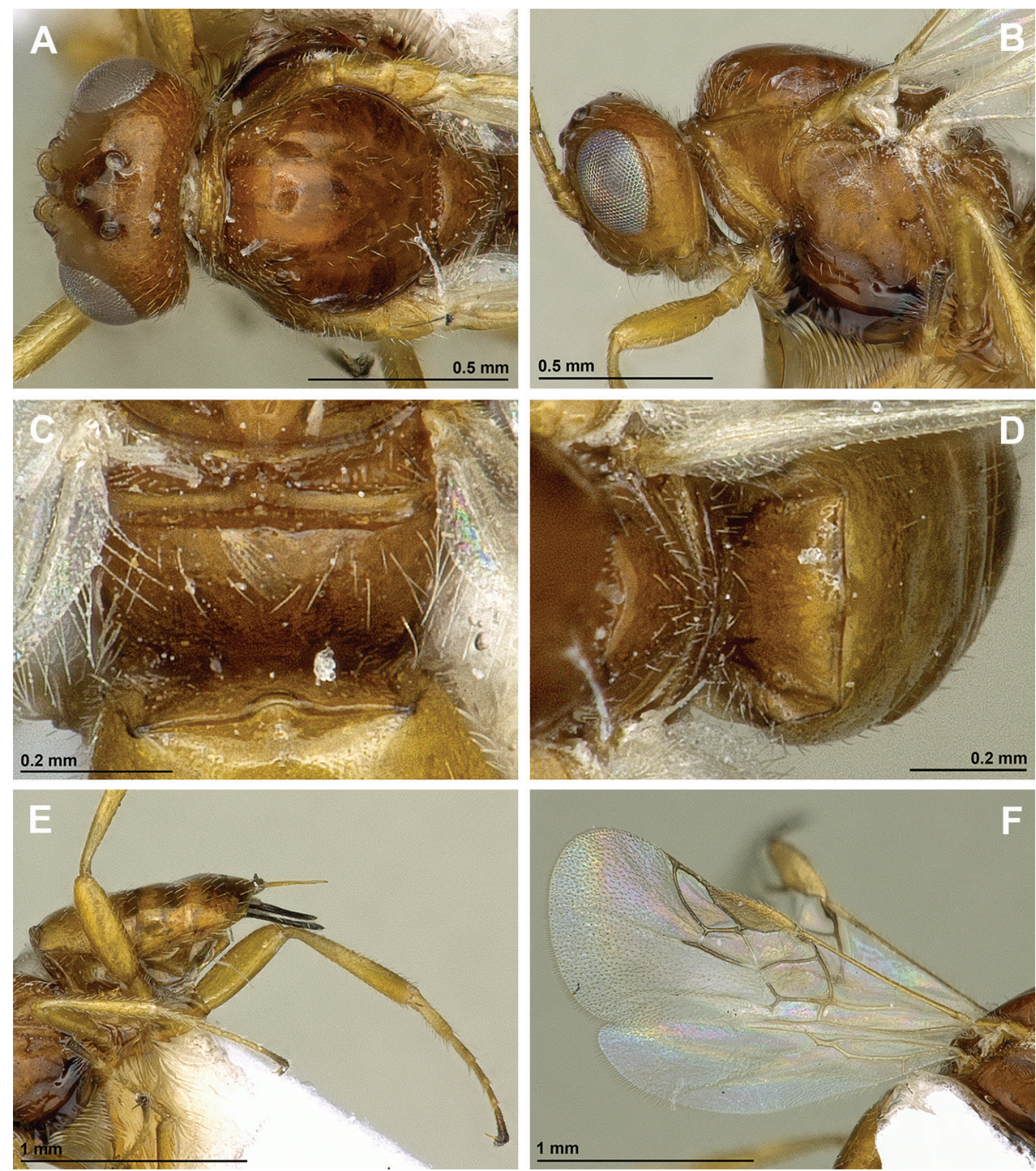

Figure 2. Hoplocrotaphus hamooniae sp. n. (female, holotype) A Head and mesoscutum, dorsal view B Head and mesosoma, lateral view C Propodeum, dorsal view D First metasomal tergite E Legs, metasoma and ovipositor, lateral view $\mathbf{F}$ Fore and hind wings.

Variation. All females have the same characters and ratios.

Male. Body $2.0 \mathrm{~mm}$; fore wing $2.0 \mathrm{~mm}$; hind wing $1.5 \mathrm{~mm}$. Antenna 21 -segmented and first flagellar segment twice as long as its maximum width. Eye in lateral view as wide as temple medially. Mesosoma in lateral view 1.4 times as long as high. First metasomal tergite 0.8 times as long as its apical width. Otherwise similar to female.

Distribution. Iran. 


\section{Hoplocrotaphus mongolicus Fischer, 1971}

Figures 3, 4

Hoplocrotaphus mongolicus Fischer, 1971: 69; Yu et al., 2016.

Material examined. 1 ㅇ (holotype), Mongolia, Central aimak, $12 \mathrm{~km} \mathrm{~S}$ von Somon Bajanbaraat, 1380 m, 13.vii.1967, Exp. Dr. Z. Kaszab (Nr. 918) (HNHM).

Comparative diagnosis. This species is similar to $H$. hamooniae sp. n.; differences between both species are described under the latter species. Moreover, this species is similar to $H$. mysteriosus Fischer, 1971 but differs from it in having the temple in lower half with distinct prominence (Fig. 3B, E, F) (without prominence in $H$. mysteriosus: Fig. 5B, C, E, F), occipital carinae not strongly separated from hypostomal carina (widely separated from hypostomal carina in $H$. mysteriosus), first flagellar segment 3.1 times as long as its maximum width (3.7 times in $H$. mysteriosus), sixth flagellar segment 1.9 times as long as its maximum width (2.9 times in $H$. mysteriosus), POL 2.8 times OD (3.8 times in H. mysteriosus), OOL 1.9 times OD (3.5 times in $H$. mysteriosus), hind femur 3.3 times as long as its maximum width (4.0 times in $H$. mysteriosus), first metasomal tergite 1.3 times as long as its apical width (0.8 times in $H$. mysteriosus), marginal cell 3.1 times as long as its maximum width (4.2 times in $H$. mysteriosus), and vein 3-SR 1.3 times as long as vein 2-SR (0.8 times in H. mysteriosus).

Re-description. Female (holotype). Head. In dorsal view, 1.9 times as wide as median length, 1.3 times as wide as mesoscutum, smooth. Eye in lateral view 1.5 times as high as wide and 1.5 times as wide as temple medially. POL 2.8 times OD; OOL 1.9 times OD. Face 2.4 times as wide as high; inner margins of eyes subparallel. Clypeus 6.6 times as wide as high, slightly curved ventrally. Temple in lower half with distinct prominence. Occipital carina ventrally not widely separated from hypostomal carinae. Mandible weakly and evenly widened towards base. Antenna 15-segmented. Scape 1.5 times as long as pedicel. First flagellar segment 3.1 times as long as its apical width, 1.1 times as long as second segment; second to fourth segments 2.1 times; fifth to 12 th 1.9 times, and 13th (apical) segment 2.1 times as long as their maximum width accordingly.

Mesosoma. In lateral view, 1.2 times as long as high. Mesoscutum 0.9 times as long as its maximum width. Posterior mesopleural furrow smooth.

Legs. Hind femur 3.3 times as long as its maximum width. Hind tibia about 7.5 times as long as its maximum subapical width, as long as hind tarsus. First segment of hind tarsus 1.6 times as long as second segment.

Wings. Length of fore wing 2.6 times its maximum width. Marginal cell 3.1 times as long as its maximum width. Vein 1-R1 0.3 times as long as pterostigma, 0.3 times as long as distance between apex of marginal cell and apex of wing. Veins $r$ shorter than pterostigma broad. Vein 3-SR 1.3 times as long as vein 2-SR. Vein m-cu postfurcal. Hind wing 4.1 times as long as its maximum width. 



Figure 3. Hoplocrotaphus mongolicus Fischer, 1971 (female, holotype) A Habitus, lateral view B Head, lateral view C Mandible D Antenna E Head, front view $\mathbf{F}$ Head and mesoscutum, dorsal view.

Metasoma. First tergite weakly widened towards apex, 1.3 times as long as its apical width. Ovipositor not exerted, 0.5 times as long as first tergite, 0.1 times as long as hind femur.

Length. Body $1.6 \mathrm{~mm}$; fore wing $2.0 \mathrm{~mm}$; hind wing $1.3 \mathrm{~mm}$.

Colour. Body, antenna, coxae and femora black. Mandible brown. Hind tibia light brown in basal part and darkened in apical. Wings hyaline. Pterostigma brown.

Male.Unknown. Distribution. Mongolia. 

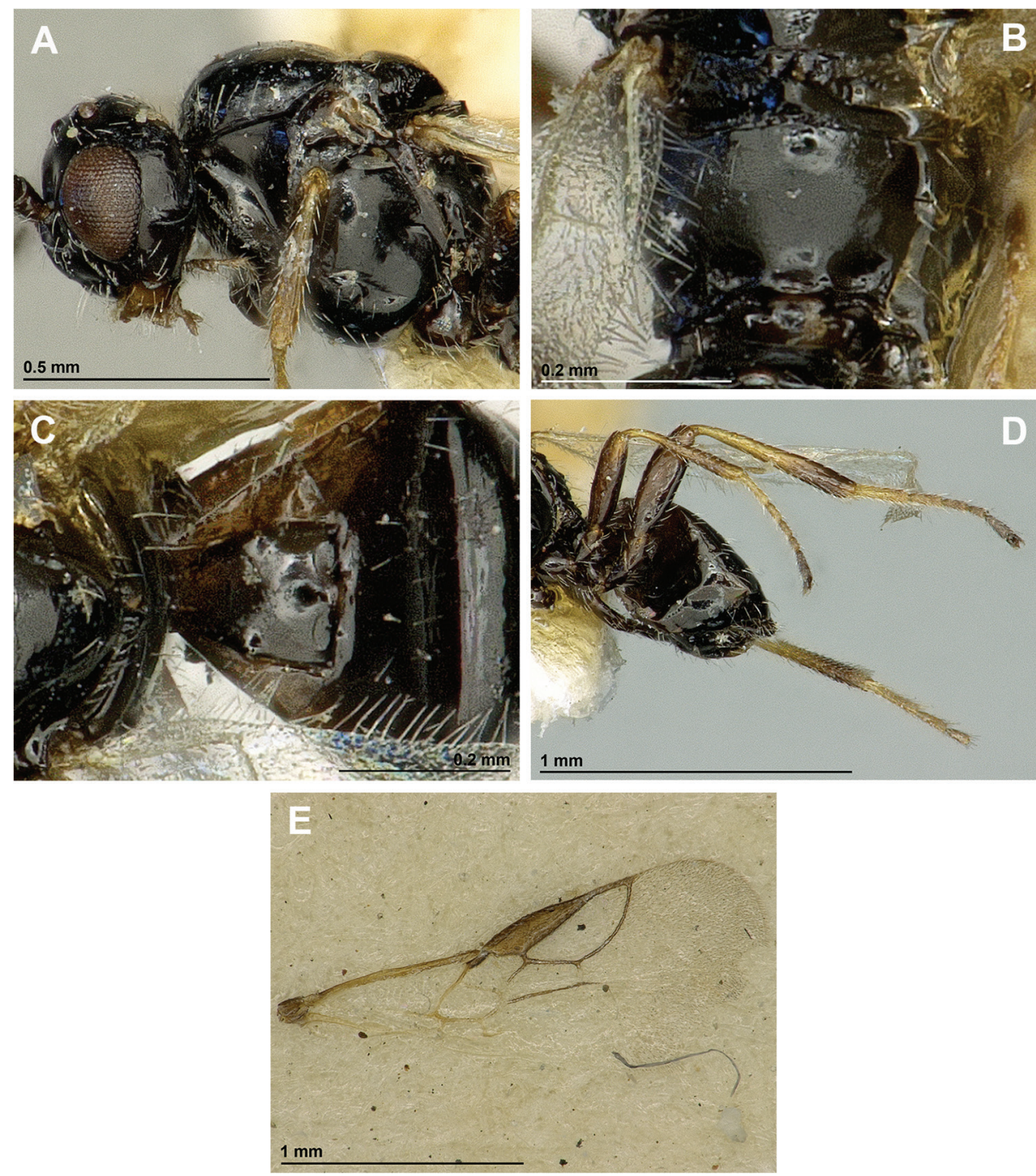

Figure 4. Hoplocrotaphus mongolicus Fischer, 1971 (female, holotype) A Head and mesosoma, lateral view B Propodeum, dorsal view C First metasomal tergite D Legs, metasoma and ovipositor, lateral view $\mathbf{E}$ Fore wing.

\section{Hoplocrotaphus mysteriosus Fischer, 1971}

Figures 5, 6

Hoplocrotaphus mysteriosus Fischer, 1971: 72; Papp, 1982: 243; Tobias and Jakimavicius, 1986: 99; Yu et al., 2016.

Material examined. $1 q$ (holotype), Mongolia, Bajan-Ölgij aimak, 20 km NNW von der Stadt Ölgij, 2100 m, 2.vii.1968, Exp. Dr. Z. Kaszab (Nr. 1054) (HNHM). 

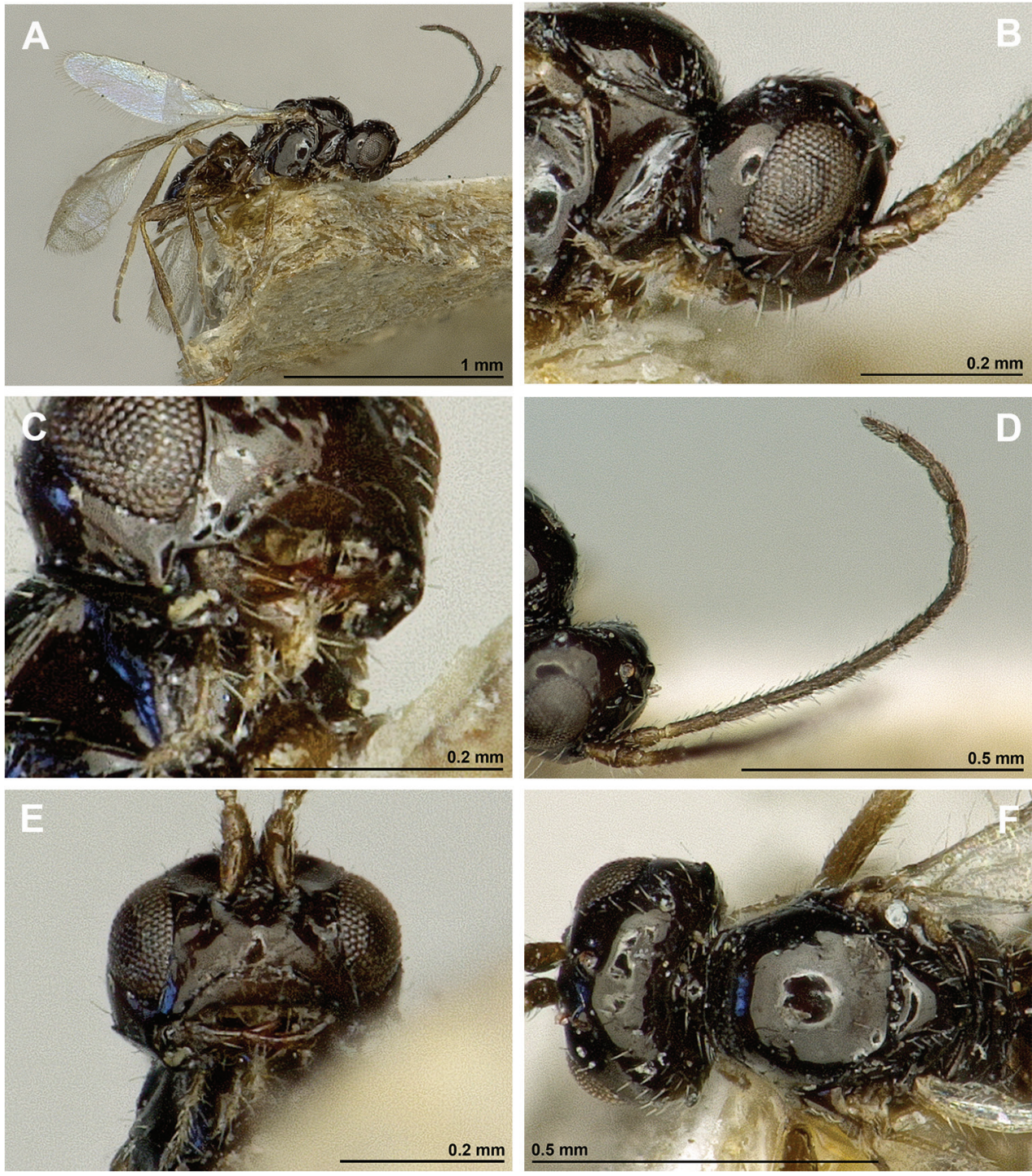

Figure 5. Hoplocrotaphus mysteriosus Fischer, 1971 (female, holotype) A Habitus, lateral view B Head, lateral view C Mandible D Antenna E Head, front view $\mathbf{F}$ Head and mesoscutum, dorsal view.

Comparative diagnosis. This species is similar to $H$. mongolicus Fischer; differences between both species are described under the latter species.

Re-description. Female (holotype).

Head. In dorsal view, 1.8 times as wide as median length, 1.3 times as wide as mesoscutum, smooth. Eye in lateral view 1.5 times as high as wide and 1.4 times as wide as temple medially. POL 3.8 times OD; OOL 3.5 times OD. Face 2.6 times as wide as high; inner margins of eyes subparallel. Clypeus 4.4 times as wide as high, slightly curved ventrally. Temple in lower half without prominence. Occipital carina ventrally 

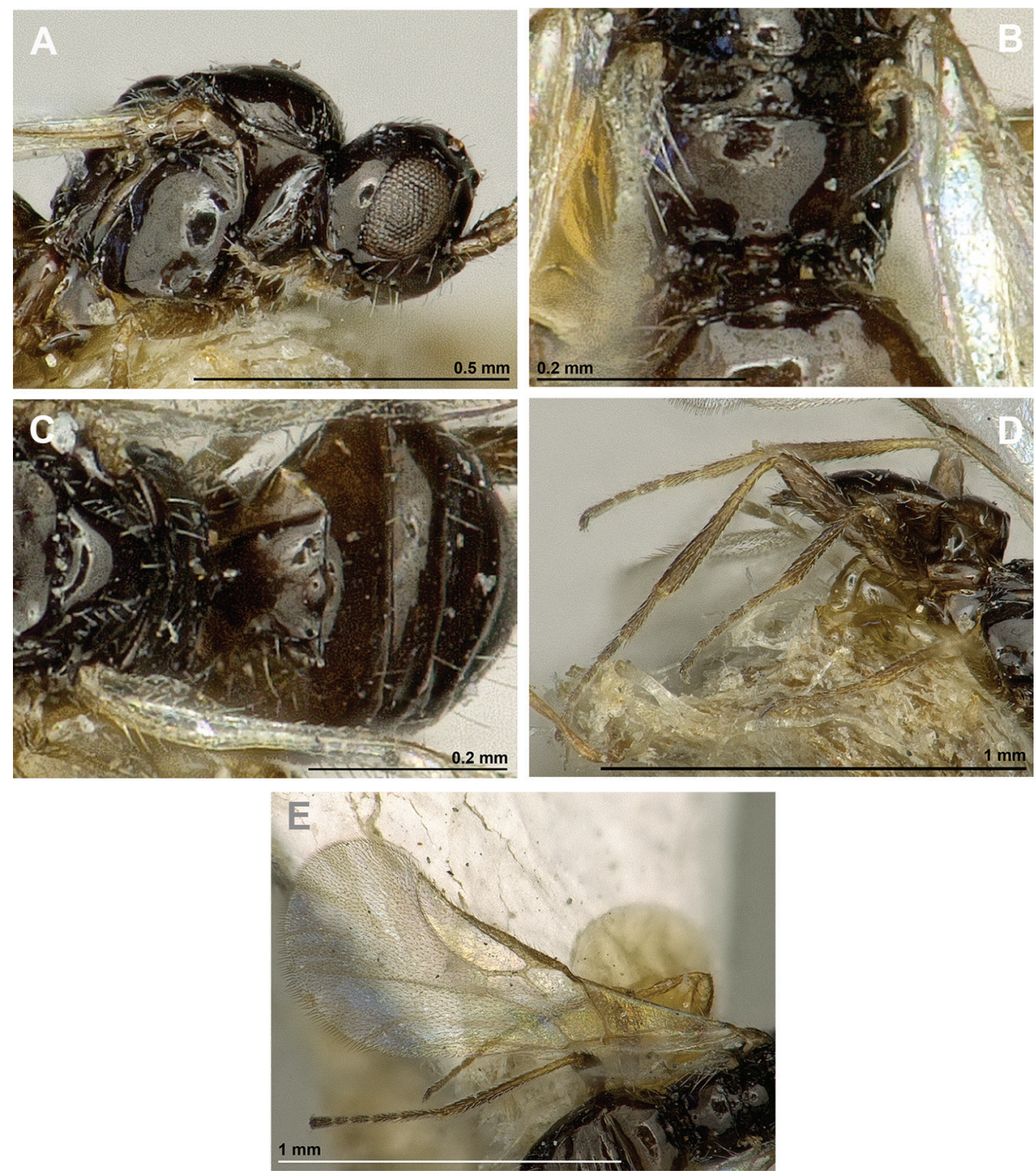

Figure 6. Hoplocrotaphus mysteriosus Fischer, 1971 (female, holotype) A Head and mesosoma, lateral view B Propodeum, dorsal view C First metasomal tergite D Legs, metasoma and ovipositor, lateral view $\mathbf{E}$ Fore wing.

rather widely separated from hypostomal carinae. Mandible weakly and evenly widened towards base. Antenna 14-segmented. Scape 1.5 times as long as pedicel. First flagellar segment 3.7 times as long as its apical width, 1.1 times as long as second segment; second and third segments 3.3-3.4 times; fourth and fifth 3.1 times; sixth to 11 th 2.9 times, and 12th (apical) segment 3.1 times as long as their maximum width accordingly.

Mesosoma. In lateral view, 1.1 times as long as high. Mesoscutum about as long as its maximum width. Posterior mesopleural furrow smooth. 
Legs. Hind femur 4.0 times as long as its maximum width. Hind tibia 8.2 times as long as its maximum subapical width, about as long as hind tarsus. First segment of hind tarsus 1.7 times as long as second segment.

Wings. Length of fore wing 3.0 times its maximum width. Marginal cell 4.2 times as long as its maximum width. Vein 1-R1 0.3 times as long as pterostigma, 0.5 times as long as distance between apex of marginal cell and apex of wing. Veins $r$ shorter than pterostigma broad. Vein 3-SR 0.8 times as long as vein 2-SR. Vein m-cu distinctly postfurcal. Hind wing 4.2 times as long as its maximum width.

Metasoma. First tergite widened towards apex, 0.8 times as long as its apical width. Ovipositor not exerted, 0.4 times as long as first tergite, 0.1 times as long as hind femur. Length. Body $1.2 \mathrm{~mm}$; fore wing $1.5 \mathrm{~mm}$; hind wing $1.1 \mathrm{~mm}$.

Colour. Body, antenna, mandible and legs dark brown. Wings hyaline. Pterostigma brown.

Male. Unknown.

Distribution. Mongolia, Romania.

\section{Hoplocrotaphus pospelovi Telenga, 1950}

Figures 7-9

Hoplocrotaphus pospelovi Telenga, 1950: 303; Tobias and Jakimavicius, 1986: 99; Yu et al., 2016.

Material examined. 1 male (lectotype, here designated), (head and antenna missing), Kazakhstan (not Uzbekistan, as in original description), "peski [sands] Koy-Temo, bl.[iz] [near] Terekshy, Priaral[skie] Karakumy [Priaral Kara-Kum], E. Luppova, 9.ix. [1]930”, “Hoplocrotaphus pospelovi g. et sp. n., N. Telenga det.” (ZISP); 1 male (?) (paralectotype), (mostly destroyed, only mesosoma and middle legs are presented on pin), Kazakhstan (not Uzbekistan, as in original description), golden circle, "peski [sands] Koylibay, M.[alye] Barsuki, Turg.[ay], Luppova, 30.vi.[1]931”, “Hoplocrotaphus pospelovi g. et sp. n., N. Telenga det." (ZISP); 1 female, 2 males, Turkmenistan, "TSSR, Repetek, V. Tobias", 24 and 25.VI.1987 (ZISP).

Comparative diagnosis. This species is similar to $H$. hamooniae sp. n., but differs by having the mandible basally with distinct ventral lobe (without ventral lobe in $H$. hamooniae), first flagellar segment 3.3 times as long as its maximum width $(2.4$ times in $H$. hamooniae), sixth flagellar segment 2.6 times as long as its maximum width (2.0-2.1 times in $H$. hamooniae), marginal cell 2.0 times as long as its maximum width (3.3 times in $H$. hamooniae), OOL 2.8 times OD (1.7 times in $H$. hamooniae), and clypeus 4.6 times as wide as high (3.7 times in H. hamooniae).

Re-description. Male (lectotype and additional specimen).

Head. In dorsal view, 2.1 times as wide as median length, 1.3 times as wide as mesoscutum, smooth. Eye in lateral view 1.4 times as high as wide and 1.3 times as wide as temple medially. POL 2.8 times OD; OOL 2.8 times OD. Face 2.4 times as wide 

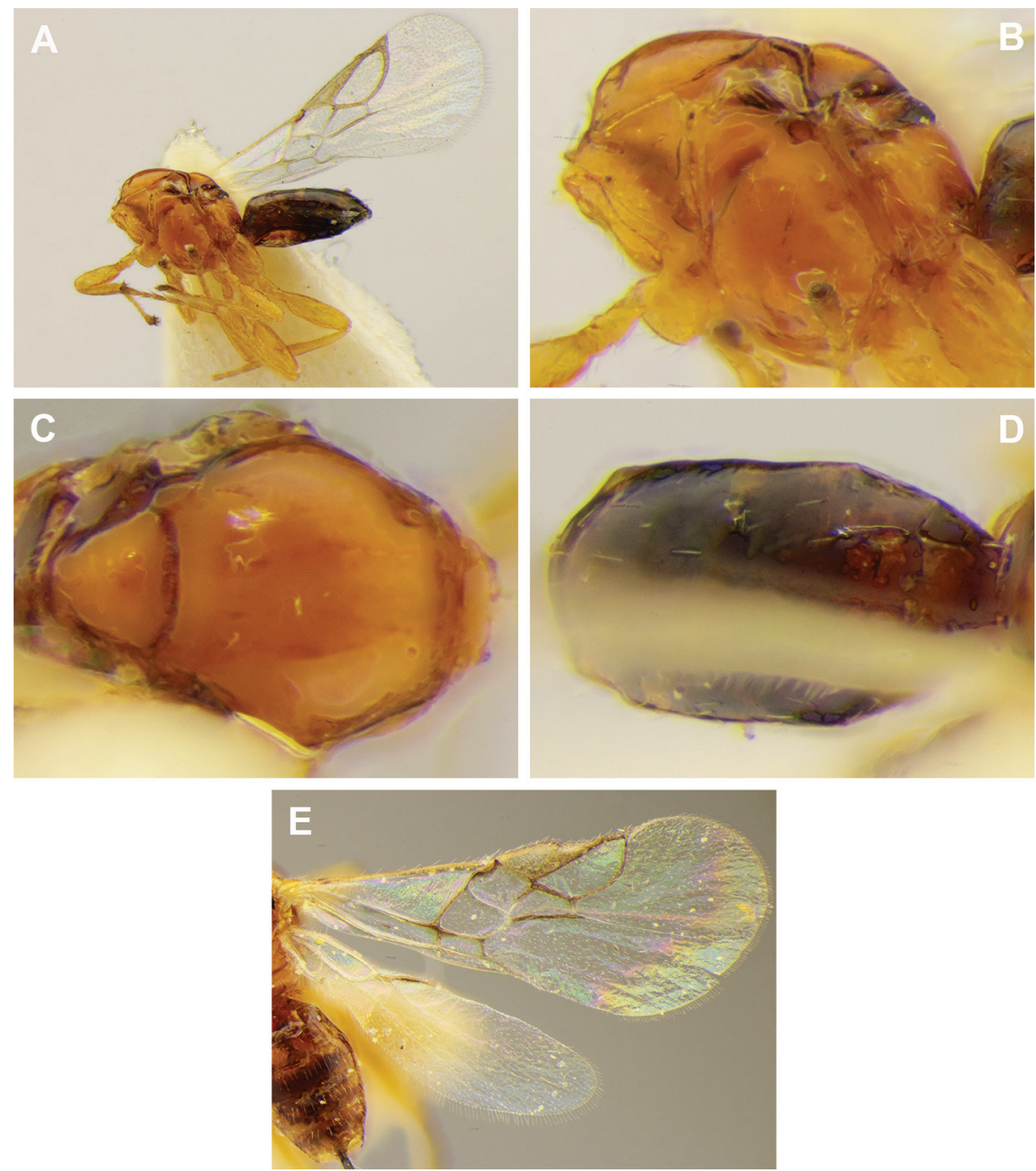

Figure 7. Hoplocrotaphus pospelovi Telenga, 1950 (A-D lectotype: male E specimen from Turkmenistan, female) A Habitus, lateral view B Mesosoma, lateral view C Mesoscutum, dorsal view D Metasoma, dorsal view $\mathbf{E}$ Fore and hind wings.

as high; inner margins of eyes subparallel. Clypeus 4.6 times as wide as high, slightly curved ventrally. Temple in lower half without prominence. Occipital carina ventrally widely separated from hypostomal carinae. Mandible basally with distinct ventral lobe. Antenna 17- segmented. Scape 1.5 times as long as pedicel. First flagellar segment 3.3 times as long as its apical width, 1.2 times as long as second segment; second segment 2.8 times; third to eighth segments $2.5-2.6$ times; ninth to $14^{\text {th }} 2.3$ times, and $15^{\text {th }}$ (apical) segment 3.3 times as long as their maximum width accordingly. 

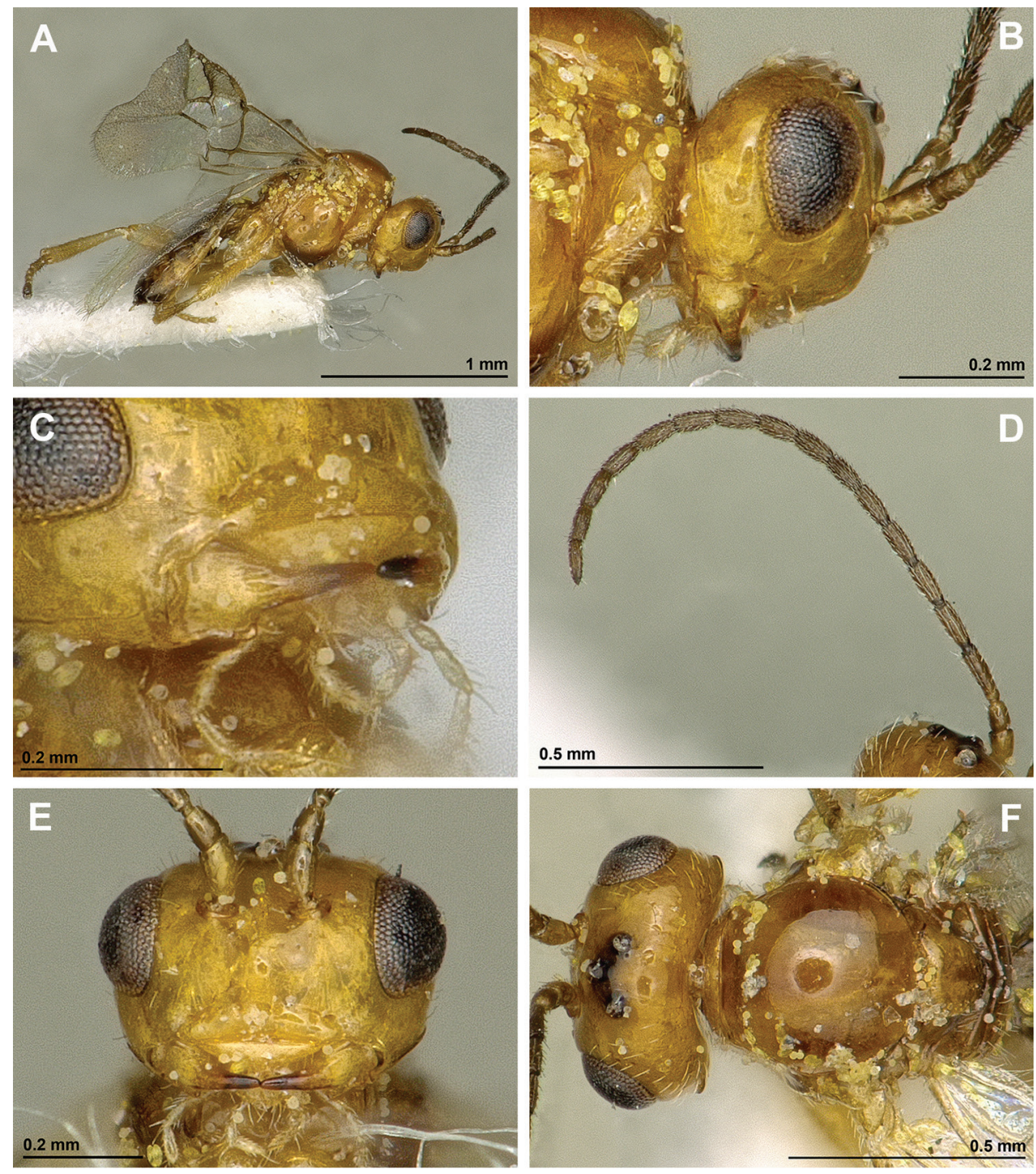

Figure 8. Hoplocrotaphus pospelovi Telenga, 1950 (male, specimen from Turkmenistan) A Habitus, lateral view B Head, lateral view C Mandible D Antenna E Head, front view F Head and mesoscutum, dorsal view.

Mesosoma. In lateral view, 1.3 times as long as high. Mesoscutum almost as long as its maximum width. Posterior mesopleural furrow smooth.

Legs. Hind femur 3.1 times as long as its maximum width. Hind tibia 6.5 times as long as its maximum subapical width, 1.1 times as long as hind tarsus. First segment of hind tarsus 1.5 times as long as second segment.

Wings. Length of fore wing 2.4 times its maximum width. Marginal cell 2.6 times as long as its maximum width. Vein 1-R1 0.25 times as long as pterostigma, 0.25 times as long as distance between apex of marginal cell and apex of wing. Veins $r$ distinctly 

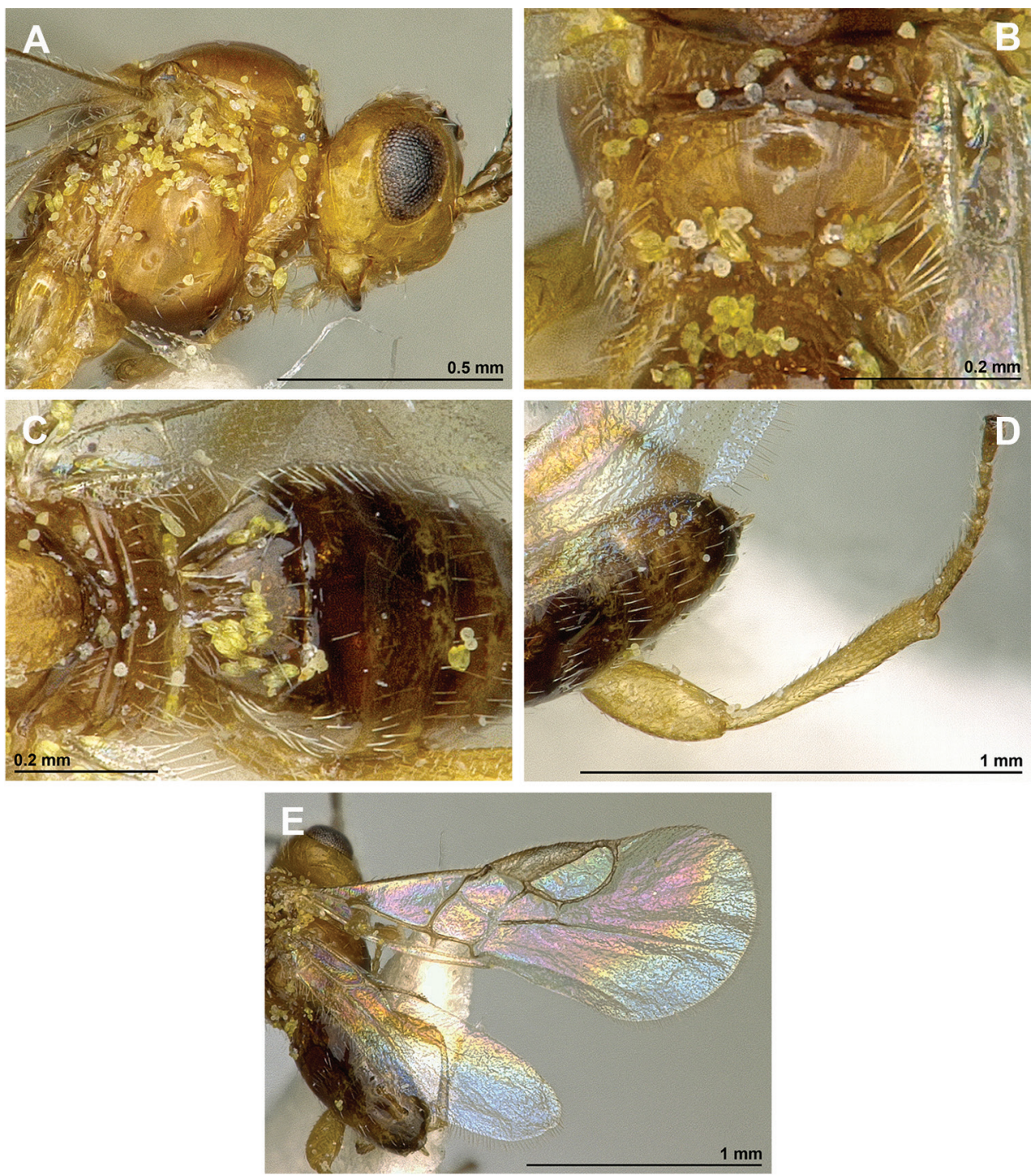

Figure 9. Hoplocrotaphus pospelovi Telenga, 1950 (male, specimen from Turkmenistan) A Head and mesosoma, lateral view B Propodeum, dorsal view C First metasomal tergite D Leg, lateral view E Fore and hind wings.

shorter than pterostigma broad. Vein 3-SR 0.9 times as long as vein 2-SR. Vein m-cu distinctly postfurcal. Hind wing 4.5 times as long as its maximum width.

Metasoma. First tergite widened towards apex, 0.9 times as long as its apical width. Length. Body $1.3 \mathrm{~mm}$; fore wing $1.5 \mathrm{~mm}$; hind wing $1.2 \mathrm{~mm}$.

Colour. Body mainly and legs light brown. Antenna brown. Metasoma dark brown. Wings hyaline. Pterostigma brown.

Variation. Body length $1.9 \mathrm{~mm}$; fore wing $1.8 \mathrm{~mm}$; hind wing $1.5 \mathrm{~mm}$. Head. In dorsal view, 1.9 times as wide as median length, 1.4 times as wide as mesoscutum. Eye 
in lateral view 1.4 times as high as wide and 1.25 times as wide as temple medially. POL 2.3 times OD; OOL 2.0 times OD. Face 1.6 times as wide as high. Clypeus 3.4 times as wide as highly. Scape 1.4 times as long as pedicel. First flagellar segment 2.7 times as long as its apical width, 1.1 times as long as second segment; second segment 2.6 times; following segments 2.4-2.5 times; and 15th (apical) segment 3.0 times as long as their maximum width accordingly. Mesosoma. In lateral view, 1.1 times as long as high. Legs. Hind femur 2.6-2.7 times as long as its maximum width. Hind tibia 5.5-5.7 times as long as its maximum subapical width. First segment of hind tarsus 1.8 times as long as second segment. Wings. Length of fore wing 2.3 times its maximum width. Marginal cell 3.1 times as long as its maximum width. Vein 1-R1 0.26-0.28 times as long as pterostigma, $0.24-0.26$ times as long as distance between apex of marginal cell and apex of wing. Hind wing 3.9-4.0 times as long as its maximum width.

Metasoma. First tergite 0.7-0.8 times as long as its apical width. Antenna dark brown.

Female. Head. In dorsal view, 2.0 times as wide as median length. Eye in lateral view 1.3 times as high as wide and 1.4 times as wide as temple medially. POL 2.0 times OD; OOL 2.3 times OD. Inner margins of eyes weakly divergent below. Clypeus 3.0 times as wide as high. Antenna more than 6-segmented (apical segments missing). Scape 1.7 times as long as pedicel. First flagellar segment 3.2 times as long as its apical width, 1.2 times as long as second segment.

Mesosoma. In lateral view, 1.1 times as long as high. Mesoscutum 1.15 times as long as its maximum width.

Legs. Hind femur 3.0 times as long as its maximum width. Hind tibia 5.3 times as long as its maximum subapical width, almost as long as hind tarsus. First segment of hind tarsus 1.8 times as long as second segment.

Wings. Length of fore wing 2.4 times its maximum width. Marginal cell 2.6 times as long as its maximum width. Vein 1-R1 0.3 times as long as pterostigma, 0.2 times as long as distance between apex of marginal cell and apex of wing. Vein 3-SR as long as vein 2-SR. Hind wing 3.6 times as long as its maximum width.

Metasoma. First tergite 0.8 times as long as its apical width. Ovipositor distinctly exerted, 1.2 times as long as first tergite, 0.6 times as long as hind femur.

Length. Body $1.9 \mathrm{~mm}$; fore wing $2.3 \mathrm{~mm}$; hind wing $1.6 \mathrm{~mm}$. Otherwise similar to male.

Distribution. Kazakhstan, Turkmenistan (new record).

\section{Key to species of the genus Hoplocrotaphus Telenga}

1 Mandible basally with rather distinct ventral lobe (Fig. 8C). Marginal cell 2.6-3.1 times as long as its maximum width (Figs 7E, 9E). - Antenna 17-segmented. Body length 1.3-2.1 mm. Body yellowish to orange. Kazakhstan, Turkmenistan.

H. pospelovi Telenga $\left(\stackrel{+}{\circlearrowright^{\top}}\right)$

- Mandible basally without ventral lobe (Figs 1D, 3C, 5C). Marginal cell 3.1-4.2 times as long as its maximum width (Figs 2F, 4E, 6E). 
2 Marginal cell of fore wing 4.2 times as long as its maximum width (Fig. 6E). Hind femur 4.0 times as long as its maximum width (Fig. 6D). First flagellar segment of $q 3.7$ times as long as its maximum width (Fig. 5D). POL 3.8 times OD. OOL 3.5 times OD (Fig. 5F). - Antenna 14-segmented. Body length $1.2 \mathrm{~mm}$. Body black to dark brown. Mongolia, Romania................................. mysteriosus Fischer $(q)$

- $\quad$ Marginal cell of fore wing 3.1-3.3 times as long as its maximum width (Figs 2F, 4E). Hind femur 3.3 times as long as its maximum width (Figs 2E, 4D). First flagellar segment of $\bigcirc 2.4-3.1$ times as long as its maximum width (Figs 1C, 3D). POL 2.3-2.8 times OD. OOL 1.7-1.9 times OD (Figs 2A, 3F). Antenna 15-21-segmented....

3 Temple in lower half with distinct subvertical prominence (Fig. 3B, C, E, F). Occipital carina ventrally not widely separated from hypostomal carinae (Fig. 3B, E). Body black to dark brown (Fig. 3A). First metasomal tergite 1.3 times as long as its apical width (Fig. 4C). Vein m-cu of fore wing distinctly postfurcal (Fig. 4E). Vein 3-SR of fore wing 1.3 times as long as vein 2-SR (Fig. 4E). Clypeus 6.6 times as wide as high (Fig. 3E). First flagellar segment of $q 3.1$ times as long as its maximum width (Fig. 3D). Antenna 15-segmented. Body length $1.6 \mathrm{~mm}$. Mongolia......... H. mongolicus Fischer $(q)$ Temple in lower half without distinct subvertical prominence (Figs 8B, E, F, 9A). Occipital carina ventrally not widely separated from hypostomal carinae (Figs 3B, C). Body light brown (Fig. 1A, B). First metasomal tergite 0.6-0.8 times as long as its apical width (Fig. 2D). Vein $\mathrm{m}-\mathrm{cu}$ of fore wing interstitial (Fig. 2F). Vein 3-SR of fore wing 0.8 times as long as vein 2-SR (Fig. 2F). Clypeus 3.7 times as wide as high (Fig. 1D). First flagellar segment of $q 2.4$ times as long as its maximum width (Fig. 1E). Antenna 20-21-segmented. Body length $2.0-2.1 \mathrm{~mm}$. Iran .....

H. hamooniae sp. n. $\left(q \bigcirc^{\lambda}\right)$

\section{Discussion}

The new species was found in association with Tamarix stricta Boiss. around Hamoon wetland during spring (April-May) and autumn (September-October). The absence during the hot summer period suggests two generations per year. These specimens were collected mainly early at night by light trapping at ground level, while no specimens were found in light traps mounted more than one meter above ground level during the whole year. The specimens share the pale body colour, but there is no morphological evidence of nocturnal activity (for example, enlarged compound eyes and ocelli: Tobias 1966), both are normal in size. It is likely that the parasitoids normally escape from the extremely hot weather during daytime by hiding among the Tamarix branches. The wing morphology has clues about flight ability and habitat preference (Quicke 2015). There is no evidence of the possible host of $H$. hamooniae sp. n. (as well as for other Hoplocrotaphus species) among the various mining dipterous larvae available on the wetland plants. 


\section{Acknowledgements}

Thanks are due to the Department of Plant Protection (University of Zabol) for support during the samplings and for supplying the laboratory facilities. Contribution by E. Rakhshani was supported by the grant UOZ-GR-9517-2, University of Zabol. The second author was supported by grants of the Russian Foundation for Basic Research (projects Nos 15-29-02466 and 16-04-00197) and the Russian State Research Project No. AAAA-A17-117030310210-3.

\section{References}

van Achterberg C (1993) Illustrated key to the subfamilies of the Braconidae (Hymenoptera: Ichneumonoidea). Zoologische Verhandelingen Leiden 283: 1-189.

van Achterberg C (2009) Can Townes type Malaise traps be improved? Some recent developments. Entomologische Berichten Amsterdam 69(4): 129-135.

Fischer M (1971) Opiinae aus der Mongolei, gesammelt von Dr. Z. Kaszab in den Jahren 1966-1968. Sitzungsberichte der Österreichischen Akademie der Wissenschaften Mathematisch-Naturwissenschaftliche Klasse. Abteilung I 179(5-6): 33-76.

Fischer M (1972) Hymenoptera, Braconidae (Opiinae I). Das Tierreich, Walter de Gruyter, Berlin, 91, 620 pp.

Fischer M (1977) Hymenoptera Braconidae (Opiinae II). (Amerika). Das Tierreich 96: 1-1001. Fischer M (1987) Hymenoptera Opiinae III - aethiopische, orientalische, australische und ozeanische Region. Das Tierreich 104: 1-734.

Papp J (1982) Taxonomical and faunistical novelities of the Opiinae in the Arctogaea (Hymenoptera, Braconidae). Annales Historico-Naturales Musei Nationalis Hungarici 74: 241-253.

Peris-Felipo FJ, Belokobylskij SA, Jiménez-Peydró R (2014) Revision of the Western Palaearctic species of the genus Dinotrema Foerster, 1862 (Hymenoptera, Braconidae, Alysiinae), Zootaxa 3885(1): 1-483. http://dx.doi.org/10.11646/zootaxa.3885.1.1

Quicke DLJ (2015) Biology, Systematics, Evolution and Ecology of Braconid and Ichneumonid Parasitoid Wasps. Wiley Blackwell, Chichester, 688 pp.

Shaw MR, Huddleston T (1991) Classification and biology of Braconidae wasps (Hymenoptera: Braconidae). Handbooks for the Identification of British Insects 7(11): 1-126.

Telenga NA (1950) Neue Arten parasitischen Hymenopteren der Familie Braconidae in der USSR. Trudy Instituta Entomologii i Fitopatologii Akademii Nauk 2: 293- 308.

Tobias VI (1966) Parasitic braconids (Hymenoptera, Braconidae) collected at the light of a quartz lamp in Turkmenia, their variability and adaptation to life in the desert. Zoologicheskiy Zhurnal 45: 1804-1814. [In Russian]

Tobias VI, Jakimavicius A (1986) Subfam. Opiinae. In: Medvedev GS (Ed.) Opredelitel' Nasekomych Evrospeiskoy Chasti SSSR, Pereponchatokrylye 3(5): 7-100. [In Russian]

Yu DS, van Achterberg C, Horstmann K (2016) Taxapad 2016, Ichneumonoidea 2015. Database on flash-drive. Ottawa, Ontario. 